\title{
Estimation of Blood Drug Concentration by LSTM network
}

\author{
Kazuya HIRAYU ${ }^{\mathrm{a},{ }^{*}}$, Hideaki KAWANO ${ }^{\mathrm{a}}$, Hideaki ORII ${ }^{\mathrm{b}}$, Yasuhiro TSUJI ${ }^{\mathrm{c}}$ \\ ${ }^{a}$ Kyushu Institute of Technology, 1-1Sensui-cho, Tobata-ku, Kitakyushu, Fukuoka, 804-8550, Japan \\ ${ }^{b}$ Fukuoka University, 8-19-1 Nanakuma, Jonan-ku, Fukuoka-si, Fukuoka, 814-0180, Japan \\ ${ }^{\mathrm{c} U n i v e r s i t y ~ o f ~ T o y a m a, ~} 2630$ Sugiya, Toyama-si, Tyama, 930-0194, Japan \\ *Corresponding Author: hirayu.kazuya599@mail.kyutech.jp
}

\begin{abstract}
Medical treatment cannot avoid the risk like side effects. In general, appropriate dosages and dosing intervals will vary among patients. The most important consideration in administration design is blood drug concentration of the patient, and it is necessary to estimate the concentration beforehand for the administration plan. However, since it is difficult to estimate personal blood drug concentration of patient, it is extremely difficult to precisely decide administration design. In this study, we construct a model to estimate personal blood drug concentration of patients using LSTM network. The proposed method is compared to models constructed in conventional studies and examined with methods. They are a statistical model and Neural Network model proposed in previous studies. As results, our model outperformed those methods.
\end{abstract}

Keywords: LSTM, medical, Neural Network

\section{Introduction}

Drug is administered for treatments as antibacterial drug. Nevertheless, to precisely design drug administration plan is difficult. Medical treatment is not able to avoid the risk of side effects and sequelae ${ }^{[1]}$. In general, appropriate dosages and dosing intervals are different among patients. The most important consideration to decide administration design is blood drug concentration of the patient, and it is necessary to predict the concentration beforehand for the administration plan. However, in the pharmacokinetics models constructed to estimate the concentration, the predicted concentration does not depend on the patient. Population pharmacokinetic analysis and Neural Network model have been proposed to estimation the personal
Table. 1. Meaning of Variables.

\begin{tabular}{|c|c|}
\hline Variable name & Meaning \\
\hline $\mathrm{DV}$ & Drug blood concentration \\
\hline Time & Elapsed time after administration \\
\hline $\mathrm{V}$ & Distribution Volume \\
\hline $\mathrm{Ka}$ & Absorption rate constant \\
\hline $\mathrm{Ke}$ & Elimination rate constant \\
\hline
\end{tabular}

concentration. However, since it is difficult to estimate personal blood drug concentration of patient, it is extremely difficult to precisely decide administration design. In this study, we construct a model to predict patient specific blood drug concentration using LSTM network as well as compare and examine with individualized models for predicting blood drug concentration constructed in the previous studies.

\section{Conventional study}

\subsection{Population Pharmacokinetics Analysis}

Fig.1 shows an example of changes in blood drug concentration of an administration. The vertical axis represents blood drug concentration and the horizontal axis represents the elapsed time after an administration. Blood drug concentration changes markedly until about first 15 hours after administration. After that, in about 30 hours or later, the blood drug concentration is close to zero. In other words, the drug disappears from inside of the blood. Thus, it is considered that the administration before three times do not affect blood drug concentrations at certain time $t$ when we estimated the blood concentration at time $t$ in repeated administrations. Therefore, the sum of three blood drug concentration before $t$ time is the blood drug concentration at $\mathrm{t}$ time. This calculation is the following 


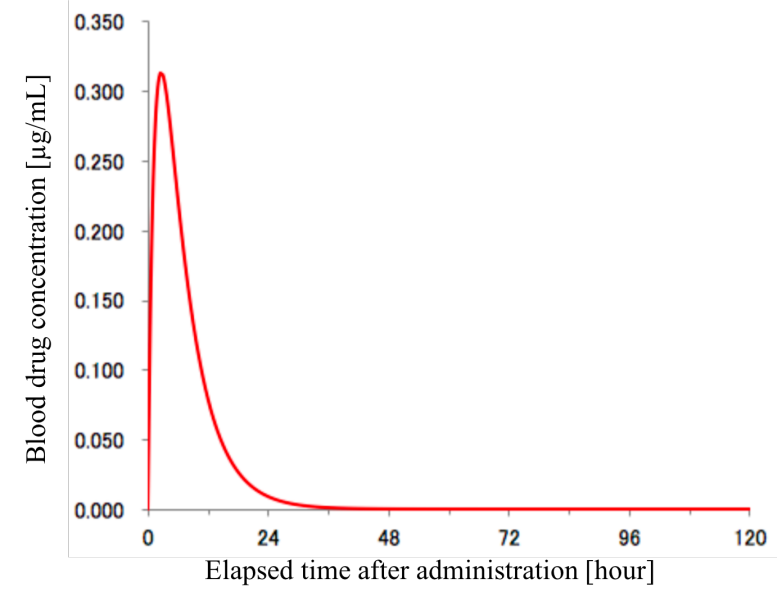

Fig 1. An example of changes in blood drug concentration after an administration.

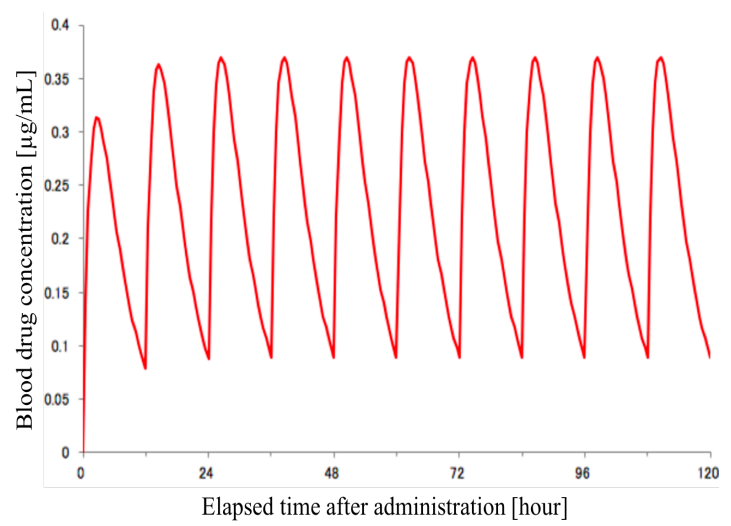

Fig. 2. An example of changes in blood drug concentration by repeated administrations.

equation.

$$
D V_{t}=\sum_{T=1}^{3} D V_{t-T}
$$

Also, Figure 2 shows an example of changes in blood drug concentration during repeated administrations.

$$
\begin{gathered}
\mathrm{DV}=\frac{\text { Does } * K_{a}}{\mathrm{~V} *\left(K_{a}-K_{e}\right)} \\
*\left(\exp \left(-K_{e} * \text { Time }\right)\right)-\left(\exp \left(-K_{a} * \text { Time }\right)\right)
\end{gathered}
$$

Equation(2) is a formula to calculate blood drug concentration. Furthermore, Table1 shows these variable's meanings. Ke can be calculated by the following equation.

$$
K_{e}=\frac{C L}{V}
$$

$C L$ that is Clearance which is used in the blood drug concentration formula, Equation(2).

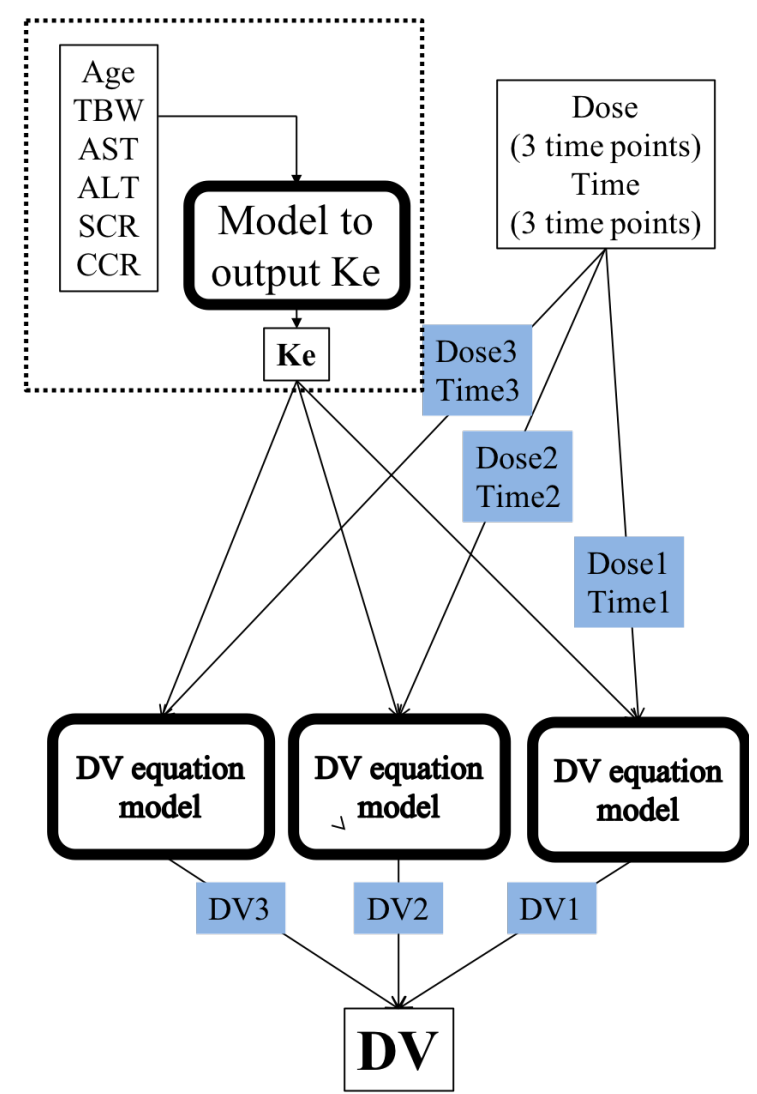

Fig. 3. Model-Driven Neural Network model diagram to estimate blood drug concentration.

According to Yasuhiro Tsuji et al, they considered that the $C L$ depends on the patient's age and total body weight. First, they decided the individualized estimation formula of $C L$ as follows.

$$
\mathrm{CL}=\theta_{1} \times\left(\frac{A g e}{60}\right)^{\theta_{2}} \times\left(\frac{T B W}{46.9}\right)^{\theta_{2}}
$$

Finally, they estimated three parameters in this equation as follows.

$$
\mathrm{CL}=17.8 \times\left(\frac{A g e}{60}\right)^{-0.269} \times\left(\frac{T B W}{46.9}\right)^{0.408}
$$

Therefore, they predicted individual blood drug concentration by this formula ${ }^{[2]}$. However, to decide Equation(4) take a lot of trouble, and it is more difficult.

\subsection{Model-Driven Neural Network}

In conventional study, Model-Driven Neural Network has been proposed to predict blood drug concentration using Neural Network ${ }^{[3]}$. In the multilayer perceptron, the structure is represented by determinant. The relation 


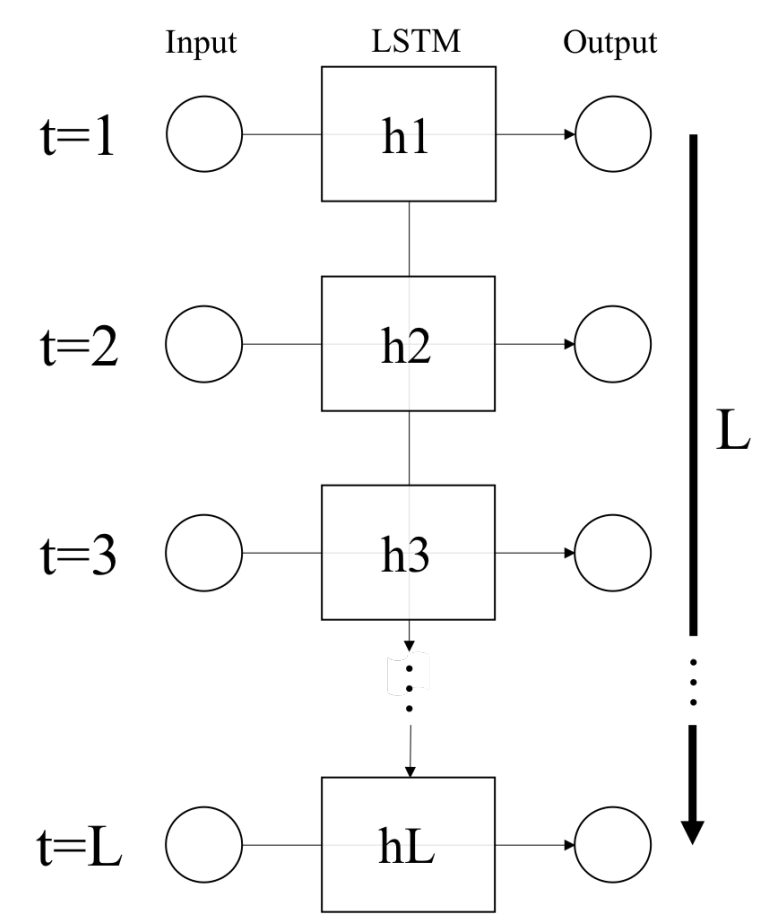

Fig. 4. Flow to use LSTM network.

between the input and output of the $j$ th neuron in a network is as follows.

$$
y_{j}=f\left(\sum_{x_{i}} w_{i j}+b_{j}\right)
$$

$x_{i}$ is the $i$ th element of the input vector to the layer. $w_{i j}$ is the weight value between $x_{i}$ and $y_{j}$, and $b_{j}$ is the bias value of the $j$ th neuron in the layer. Therefore, in the neural network, if the number of neurons in a layer is $M$, the output vector $y$ is $\left[y_{1}, y_{2}, \cdots, y_{M}\right]$, and the input vector $x$ is $\left[x_{1}, x_{2}, \cdots, x_{M}\right]$. They are calculated by the following equation.

$$
\mathrm{y}=\left[\begin{array}{ccc}
w_{11} & \cdots & w_{1 N} \\
\vdots & \ddots & \vdots \\
w_{M 1} & \cdots & w_{M N}
\end{array}\right]\left[\begin{array}{c}
x_{1} \\
\vdots \\
x_{N}
\end{array}\right]+\left[\begin{array}{c}
b_{1} \\
\vdots \\
b_{M}
\end{array}\right]
$$

In that model, there are 2 processes to train. That structure is shown in Fig.3. Ke in Equation(2) is regarded as important item to estimate personal blood drug concentration. Thus, a model to predict $K e$ was constructed. The model needs to do backpropagation from blood drug concentration since $K e$ cannot be observed. Equation(2) was trained at first and set that trained model in before output of whole structure. Finally, a model to output $\mathrm{Ke}$ was trained while fixing parameters of equation model.

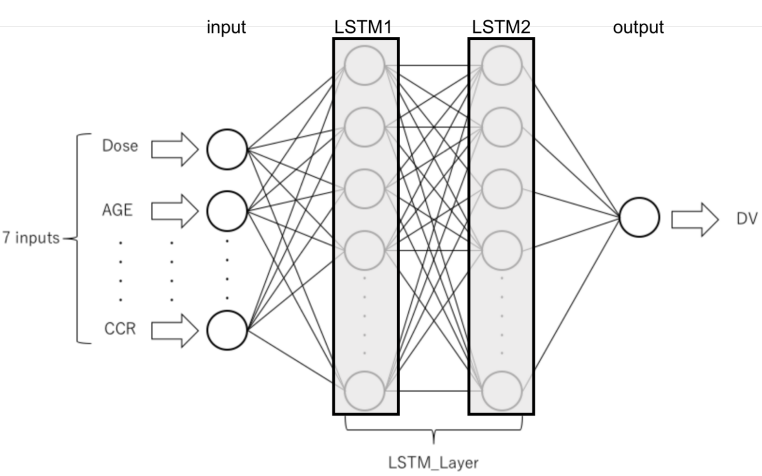

Fig. 5. Proposed structure used LSTM layers.

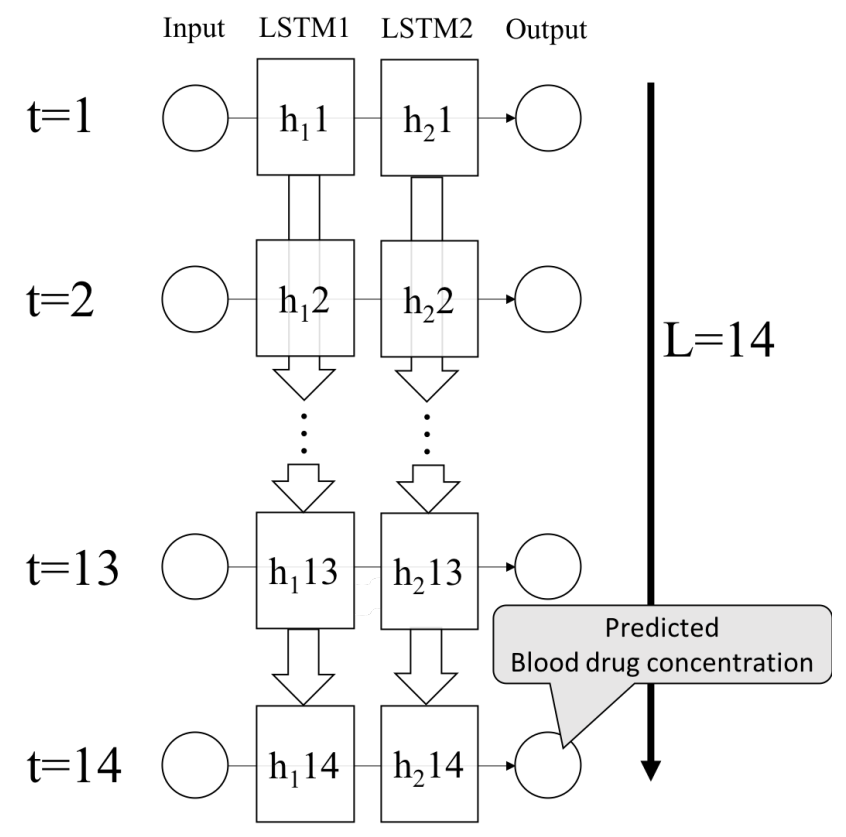

Fig. 6. Flow of proposed model.

\section{Proposed Method}

\subsection{LSTM}

Long short-term memory (LSTM) network is used in this study. LSTM is suitable for handling time series data and improved Recurrent Neural Network $(\mathrm{RNN})^{[4]}$. Unless LSTM layer is reset, that can hold previous time information alike Fig.4. Compared to RNN, LSTM can deal with the exploding and vanishing gradient problem at training of RNN ${ }^{[5]}$. LSTM layer has a cell, an input gate, an output gate and a forget gate. Also, there are some parameters at each gate. When training, these are updated like normal parameters, weight and bias. 


\subsection{Proposed Structure}

The used data in this study is time series since that has elapsed time data after administration. Thus, in my study, LSTM network is adopted to predict Blood drug concentration. Figure 5 shows the purposed structure used LSTM. These input items are Dose, Age, TBW and data for evaluation of liver function. Detail of the items is presented in Section 4.1. Also, the output of this network is only blood drug concentration and this network has 2 hidden layers. Regarding to these LSTM layers, the input at one time is data for 14 time series. In the future, that number is needed to reduce possible because that number is tentatively decided.

Figure 6 shows flow of purposed structure when 14 time series data are input. By inputting of first 13 time series data, the value in LSTM layer can be calculated and updated. In the other word, the timing information of 13 time series data can be hold before the data at 14 time is input. Finally, when last 14 time series data are input to the network, blood drug concentration can be output from that whole network using the value updated in LSTM layer.

In general, training of Neural Network is in need of numerous datasets. However, the used data in this study is observed from only 36 patients. That number is 89 and insufficient for general training of Neural Network. Therefore, Transfer Learning is adopted to compensate the few data in my study. Transfer Learning is a method to help purposed training utilizing models trained in other cases.

At first, the proposed model is trained using data, which is similar to data in considering case. Those trained parameters are set as the initial value of network of purpose before purposed training. Thus, better results are gotten at training using real data. [13] In this study, based on Transfer Learning, Equation(5) that is constructed by conventional study is utilized to training of advance. First the data for training of advance is generated by Equation(5) and proposed network is trained using that data. Next, the network is trained with real data. In other word, that do finetuning ${ }^{[6]}$.

\section{Experiment}

\subsection{Datasets}

This section describes the data that is used in training of LSTM network. The data is clinical data which was

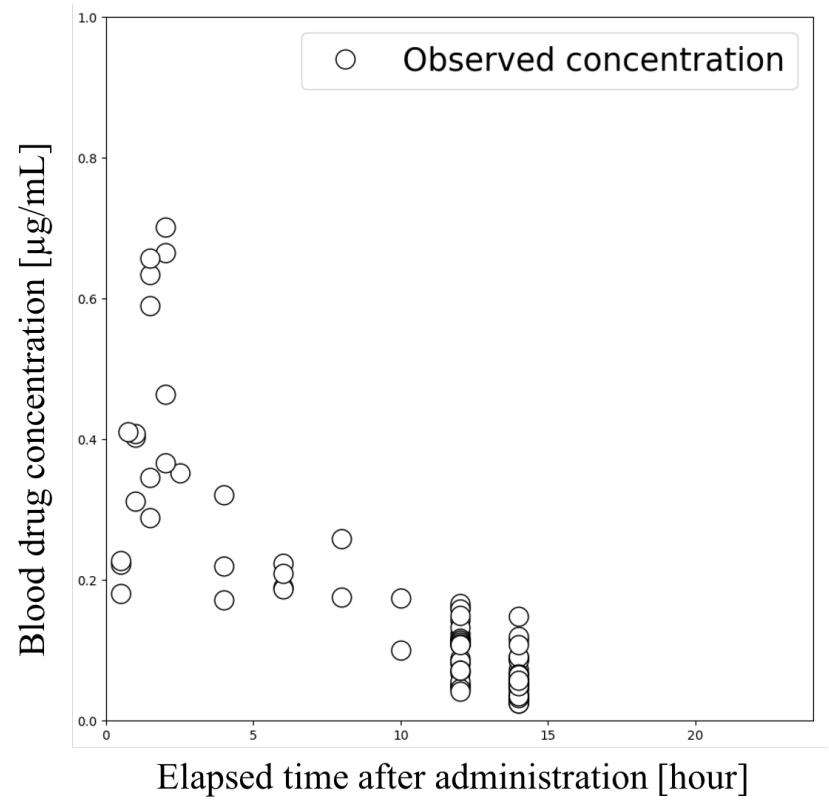

Fig. 7. Distribution of observed data.

Table. 2. Data of a certain patient.

\begin{tabular}{|c|c|c|c|c|c|c|c|}
\hline ID & Date & Time & Dose & Age & $\cdots$ & CCR & DV \\
\hline 1 & $5 / 21$ & $18: 00$ & 50 & 65 & & 79.2 & \\
1 & $5 / 22$ & $8: 30$ & 50 & 65 & & 91.5 & \\
1 & $5 / 22$ & $18: 00$ & 50 & 65 & & 91.5 & \\
1 & $5 / 23$ & $8: 30$ & 50 & 65 & & 91.5 & \\
1 & $5 / 23$ & $18: 00$ & 50 & 65 & $\cdots$ & 91.5 & \\
1 & $5 / 24$ & $8: 30$ & 50 & 65 & & 91.5 & \\
1 & $5 / 24$ & $18: 00$ & 50 & 65 & & 91.5 & \\
1 & $5 / 25$ & $8: 30$ & 50 & 65 & & 91.5 & \\
1 & $5 / 25$ & $18: 00$ & 50 & 65 & & 91.5 & \\
1 & $5 / 26$ & $6: 00$ & 50 & 65 & & 73.5 & 115 \\
\hline
\end{tabular}

Table. 3. Formal name of item for liver function.

\begin{tabular}{|c|c|}
\hline Variable name & Formal name \\
\hline AST & Aspartate Aminotransferase \\
\hline ALT & Alanine Transaminase \\
\hline SCR & Serum Creatinine \\
\hline CCR & Creatinine Clearance \\
\hline
\end{tabular}


Table. 4. Range of each item.

\begin{tabular}{|c|c|}
\hline Input Items & Range \\
\hline Dose & $25-100$ \\
\hline Age & $15-95$ \\
\hline TBW & $30-100$ \\
\hline AST & $8.0-130.0$ \\
\hline ALS & $7.0-380.0$ \\
\hline SCR & $0.20-4.50$ \\
\hline CCR & $15.0-205.0$ \\
\hline
\end{tabular}

Table. 5. Data to train and test.

\begin{tabular}{|c|c|}
\hline ID of training data & ID of test data \\
\hline $2-36$ & 1 \\
\hline $1,3-36$ & 2 \\
\hline $1,2,4-36$ & 3 \\
\hline$\cdots$ & $\cdots$ \\
\hline $1-35$ & 36 \\
\hline
\end{tabular}

observed from 36 patients (10 males and 26 females) and that distribution is shown in Fig.7. Cyclosporine had been administered to them for the treatment of connective tissue diseases regularly between April 2009 and March 2014 at Sasebo Chuo Hospital, Nagasaki, Japan. Also, that one had been administered twice a day and administration interval is 12 hours. Table 2 shows one of data of a certain patient which received cyclosporine. The item named $I D$ has 1-36 numbers and that mean identification number for the patients. The observed data used at training in LSTM network is Dose, Age, TBW, AST, ALT, SCR and CCR. TBW is Total Body Weight of each patient. Moreover, $A S T, A L T$, $S C R$ and $C C R$ are values of liver function like Table 3.

The datasets for training of advance are generated using random numbers for input data and blood drug concentration that was calculated by Equation(5). The range of each value is shown at Table 4. The blood drug concentration which is target data at training in LSTM is calculated by Equation(1). In other word, that target data is generated per 14 time series data. Furthermore, the number of the datasets for training of advance is 1000 .

\subsection{Training}

In this training, the number of units of hidden layers is decided tentatively. Data observed is only 36 numbers. Thus, in order to evaluate versatility of the trained model, 35 data

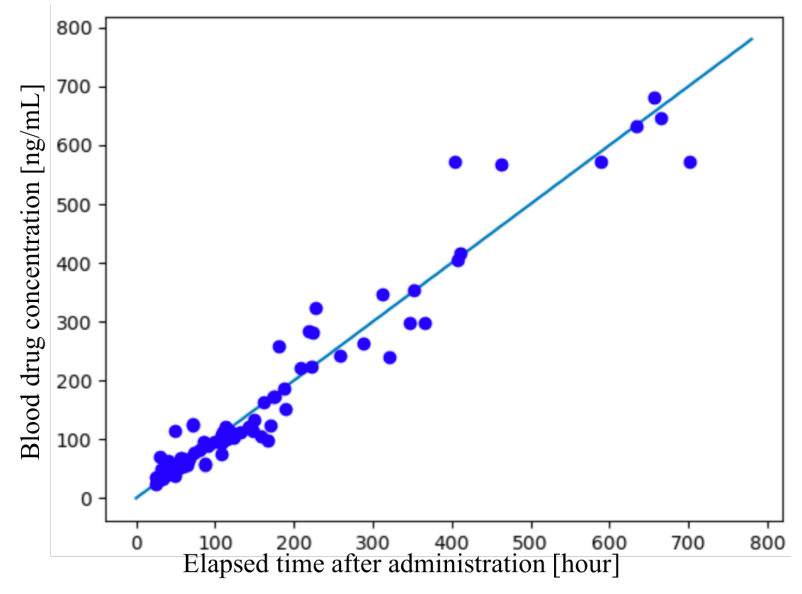

(a) Layer : 7-20-15-1.

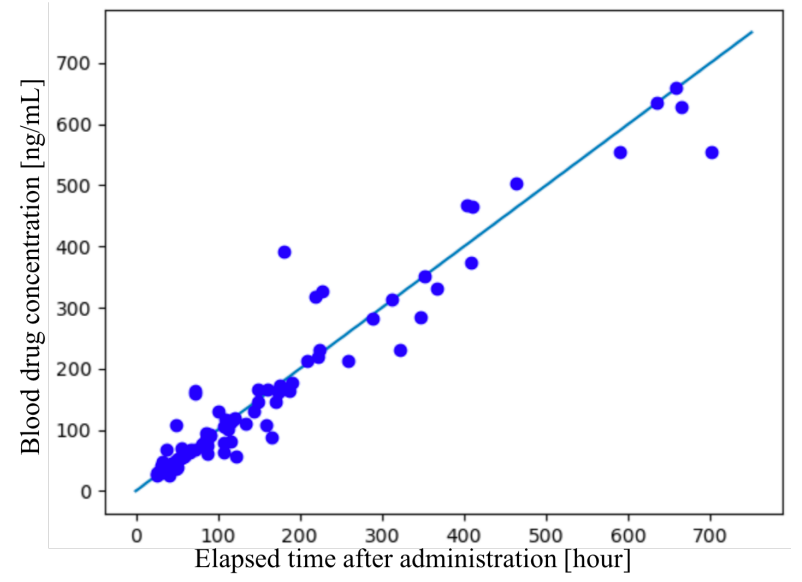

(b) Layer : 7-30-25-1.

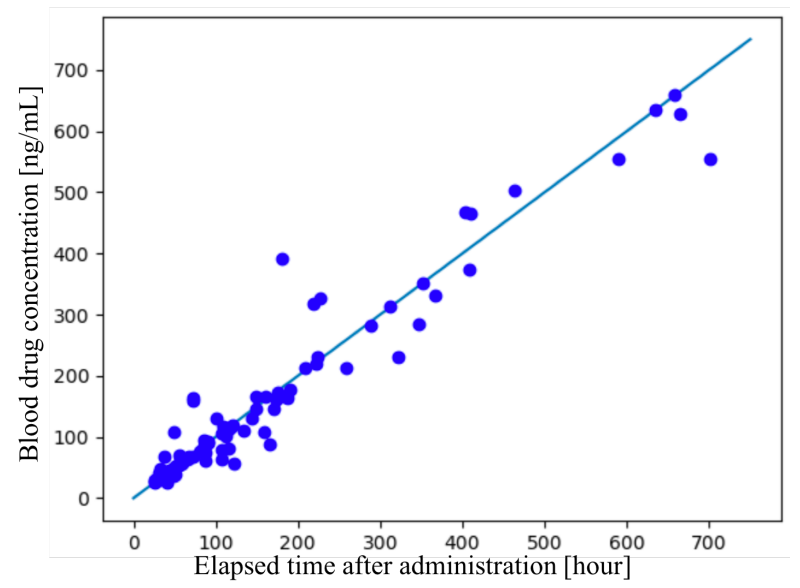

(c) Layer : 7-40-35-1.

Fig. 8. Graph of RMSE of prediction used test data in each model.

are used to train and other data is used for test (35-1). Training is done with all combinations 35-1 like Table 5, that is, 36 models is constructed finally. Therefore, RMSE of test of all model is evaluated like following formula if $t_{i}$ 
is desired data and $y_{i}$ is predicted data.

$$
R M S E=\sqrt{\frac{1}{N} \sum_{i=1}^{N}\left(t_{i}-y_{i}\right)^{2}}
$$

\subsection{Evaluation}

Table 7 shows RMSE of prediction of data for training and test by each model. Proposed network, LSTM network, is better than 2 conventional studies in terms of RMSE of testing. Also, in this study, the number of unit in hidden1hidden2 layer was tentatively 20-15, 30-25, 40-35. In any cases, test loss was able to be around 20 or under as Table 8. Moreover, compared to training loss, test loss is close in that. It was found that Transfer Learning can make training for purpose efficient.

\section{Conclusions}

The purpose of this study is to exceed the accuracy of the $D V$ estimation model constructed in previous studies. We attempted to construct LSTM network model to estimate blood drug concentration since the type of data is time series. As a result, the trained model in this study is better than Neural Network model and Pharmacokinetics model. Observed data for training is only 89 numbers, 36 patients, though, Transfer Learning was able to compensate the few data. In general, there are few clinical data. Thus, Transfer Learning is efficient to medical field. Furthermore, LSTM is suitable for handling clinical data because almost all the data is time series type and time data is important in medical field.

However, in this study, structure shape was decided tentatively and trained. If that is considered and can be set suitable structure, proposed model can be getting more accuracy. For example, the number of LSTM layer and unit of hidden layer etc. should be considered.

In the future, our proposed model should be improved. Additionally, we will attempt to propose Ladder Network. This method is one of excellent semi-supervised training. LSTM network and Ladder network will be helpful in medical research.

\section{Acknowledgment}

I would like to thank JSPS Grant-in-Aid for Scientific Research 17K00383, Casio Science Promotion Foundation, and Telecom Advanced Technology Support Center.
Table. 7. RMSE of prediction used training data and test data in each model.

\begin{tabular}{|c|c|c|}
\hline $\begin{array}{c}\text { Unit Number } \\
\text { (input-hidden(s)-output) }\end{array}$ & $\begin{array}{c}\text { Training } \\
\text { Loss } \\
{[\mathbf{n g} / \mathbf{m L} \mathbf{L}]}\end{array}$ & $\begin{array}{c}\text { Test } \\
\text { Loss } \\
{[\mathbf{n g} / \mathbf{m L} \mathbf{l}}\end{array}$ \\
\hline $7-20-15-1$ & 23.5 & 18.9 \\
\hline $7-30-25-1$ & 21.5 & 21.6 \\
\hline $7-40-35-1$ & 21.6 & 24.2 \\
\hline
\end{tabular}

Table. 8. RMSE of prediction used test data in each model and 2 conventional models.

\begin{tabular}{|c|c|c|}
\hline \multicolumn{2}{|c|}{ Method } & $\begin{array}{c}\text { RMSE } \\
{[\mathbf{n g} / \mathbf{m L}]}\end{array}$ \\
\hline \multicolumn{2}{|c|}{ Pharmacokinetics model } & 41.1 \\
\hline \multicolumn{2}{|c|}{ Model-Driven Neural Network } & 33.6 \\
\hline \multirow{2}{*}{ LSTM } & $7-20-15-1$ & 18.9 \\
\cline { 2 - 3 } Network & $7-30-25-1$ & 21.6 \\
\cline { 2 - 3 } & $7-40-35-1$ & 24.2 \\
\hline
\end{tabular}

\section{References}

(1) Hiroyasu Ogata, Takahiro Tanigawa, Mari Shiomi, Shinichi Tsuchiwata and Kanji Komatsu: "Population PK-PD analays as pharmaceu- tical development tool (in Japanese)", asakura-syoten7-8, 2010

(2) Taro Yasuhiro Tsuji, Nozomi Iwanaga, Emi Suemoto, Yoichi Hiraki, Yukio Ota, Hidefumi Kasai, Eiji Yukawa, Yukitaka Ueki, and Hideto To: "Population Pharmacokinetic Approach to the Use of Low Dose Cyclosporine in Patients with Connective Tissue Diseases", Biol. Pharm. Bull. , Vol38.No9, 2015

(3) Kazuya Hirayu, Shota Tanabata, Hideaki Kawano Yasuhiro Tsuji: "Estimation of Blood Drug Concentration by Fusing Pharmacological Model and Computational Intelligence", In Proceedings of 12th International Conference on Innovative Computing, Information and Control (ICICIC2017), ICICIC2017SS17-01, pp139, 2017.

(4) Sepp Hochreiter and Jurgen Schmidhuber: "Long short-term memory", In Neural Computation, 2013.

(5) Razvan Pascanu, Tomas Mikolov and Yoshua Bengio: "On the difficulty of training recurrent neural networks", In Proceedings of ICML, 2013.

(6) Sinno J. Pan and Qiang Yang: "A survey on transfer learning", IEEE Trans. on Knowledge and Data Engineering, Vol.22, No.10, pp.1345-1359, Oct 2010. 